\title{
Pressure gradient effect in natural convection boundary layers
}

\author{
F. J. Higuera A. Liñán
}

\begin{abstract}
The high Grashof number laminar natural convection flow around the lower stagnation point of a symmetric bowl-shaped heated body is analyzed. A region is identified where the direct effect on the flow of the component of the buoyancy force tangential to the body surface is comparable to the indirect effect of the component normal to the surface, which acts through the gradient of the nonuniform pressure that it induces in the boundary layer. Analysis of this region provides a description of the evolution of the flow from a pressure-gradient dominated regime to a buoyancy dominated regime. Numerical results are presented for the flows above and below heated power-law body shapes, and the upstream propagation of small perturbations to the stationary flow is discussed. An asymptotic analysis is carried out for the flow below nearly flat horizontal bodies, for which the change from pressure-gradient-driven to buoyancy-driven flow occurs very rapidly in a short region. The influence of body edges located in the region of interest is also discussed.
\end{abstract}

\section{INTRODUCTION}

The natural convection flow generated by heated bodies is confined, for large Grashof numbers, to thin boundary layers around the body and to ascending plumes. The flow in the boundary layers is primarily induced by the component of the buoyancy force tangential to the surface. The component of the buoyancy force normal to the surface gives rise to a nonuniform distribution of reduced pressure $p^{\prime}=p+\rho_{\infty} g z$ ( $z$ being upward vertical distance, $\rho_{\infty}$ the density of the fluid far from the body, and $p$ the static pressure), whose gradient along the surface is, however, negligible compared with the tangential component of the buoyancy force outside the bottom region of the body. Here, if the angle of the surface to the horizontal is zero or small, the tangential component of the buoyancy force is small and we may need to take into account the influence of the reduced pressure gradient on the flow.

A large amount of work, beginning with the classical work of Hermann ${ }^{1}$ for the flow around a heated circular cylinder, has been devoted to the analysis of the natural convection flow around heated bodies, with the assumption that the effect of the normal component of the buoyancy force can be neglected. With this assumption, the boundary layer equations are parabolic and the integration can be carried out starting at the lower region, with the local self-similar solution of the equations. Since no self-similar solution of the boundary layer equations exists in general for the whole body, several approximate techniques have been applied, including integral methods, series expansions, and numerical methods. Thus, Chiang and Kaye ${ }^{2}$ carried out an analysis in terms of Blasius series, and Saville and Churchill ${ }^{3}$ used Goertler series that, because of its faster rate of convergence, allow the solution to be extended farther away from the stagnation point. Muntasser and Mulligan ${ }^{4}$ obtained a solution of the boundary layer equations by means of the so-called local nonsimilarity approximation. A review and comparisons with other results obtained without the boundary layer approximation can be found in Gebhart et al. ${ }^{5}$ For bodies of finite radius of cur- vature, the neglect of the pressure gradient does not result in an error near the lower stagnation point in the limit of infinite Grashof numbers, because the scaled boundary layer thickness is finite and constant there and the effect of the pressure gradient, evaluated with the buoyancy-driven solution, is then negligible. On the other hand, while analogous solutions exist for the flow above a concave body, these are seldom if ever observed, owing to their instability.

Self-similar solutions exist for bodies with appropriate shapes. Thus, Schuh ${ }^{6}$ considered boundary layers of constant thickness, while Merk and Prins ${ }^{7}$ investigated general conditions for self-similarity below two-dimensional and axisymmetric bodies in the absence of the pressure gradient effect. Under the same assumption, Braun et $a l^{8}$ found a family of self-similar solutions for body shapes ranging from sharp edged to finite curvature and to nearly horizontal surfaces. Their solutions lead to an infinite boundary layer thickness at the lowest point of surfaces with locally infinite curvature radius, and to zero boundary layer thickness for surfaces of vanishing local curvature radius. All these solutions are applicable only at a certain distance from the lowest point.

The nonuniform reduced pressure distribution across the boundary layer results from a hydrostatic balance of the pressure gradient and the normal component of the buoyancy force, because the flow is nearly parallel to the body surface and accelerations normal to the surface are negligible. This balance leads to a high pressure in the regions below a heated surface where the fluid temperature is high or the boundary layer is thick, whereas the opposite occurs above a heated surface. Thus the fluid is pushed tangentially to the surface away from (respectively toward) these regions. Under this driving force, the flow below a horizontal plate acquires a motion toward the edge, and that above a horizontal plate moves from the edge (where the well-known Stewartson ${ }^{9}$ self-similar solution holds locally) toward the center. The effect of the self-generated pressure gradient renders the solution of the 
boundary layer problem dependent on the downstream conditions. This has been long recognized for the outward bound flow below a horizontal heated surface, for which even approximate integral methods require a boundary condition at the edge of the surface, in addition to a regularity condition at the center. A variety of conditions at the edge has been proposed. Wagner ${ }^{10}$ assumed that the boundary layer thickness is zero there, which is appropriate only for the flow above a heated surface; Singh and Birkebak $^{11}$ adjusted the movable singularity appearing in the equations of their integral method to make it coincide with the edge; Clifton and Chapman ${ }^{12}$ used an adaptation of the idea of critical conditions from open channel hydraulics. The structure of the boundary layer near the edge has been analyzed elsewhere, ${ }^{13}$ showing that the boundary layer solution develops a singularity at the edge, where the flow becomes critical, in the sense that upstream propagation of small perturbations ceases to be possible. Knowing the nature of this singularity, an appropriate boundary condition was devised to describe the flow upstream the edge. The same singularity was found independently by Daniels ${ }^{14}$ in the analysis of the horizontal boundary layers in a thermal cavity flow driven by lateral heating.

Pera and Gebhart ${ }^{15}$ and Jones ${ }^{16}$ analyzed the incipient effect of the tangential component of buoyancy on the pressure gradient-dominated boundary layer near the edge of an upward facing heated semi-infinite plate slightly inclined to the horizontal, and Ackroyd ${ }^{17}$ extended this analysis to include variable flow properties. Jones ${ }^{16}$ integrated numerically the boundary layer equations farther away from the edge for both upward and downward inclined plates. In the first case, he described the transition from the pressure gradient dominated to the buoyancy dominated regime, while, in the second case, he found a regular separation at a certain distance from the edge, and was able to continue the numerical integration through the region of reverse flow. Irrespective of the physical meaning to be assigned to the reverse flow, the possibility of continuing the marching integration points out that some selfadjusting mechanism is at work, which would not occur in a parabolic boundary layer. Jones attributed this feature to the fact that the pressure gradient is not given in advance, being determined by the flow itself. Thus the "elliptic" character of the problem underlies this solution, even if it can be obtained by a marching technique.

In this paper we analyze the boundary layer flows above and below the surface of a heated bowl-shaped body in the region around the lowest point of the surface where the changes in surface height and the boundary layer thickness are of the same order and, therefore, the effects of the pressure gradient and of the component of buoyancy tangential to the surface are equally important. Formally, for high Grashof numbers, this region has a size much smaller than the characteristic radius of curvature of the body, but it can be quite appreciable for values of the Grashof number of practical interest, the more so for flat-bottomed bodies [cf. the estimate (6) below]. The shape of the body is approximated by a power law in the neighborhood of the lower stagnation point. Numerical results are presented for different powers, to show the difference with the heat flux obtained when the pressure gradient is neglected. The evolution of small perturbations superposed on the stationary flow is discussed, pointing out that upstream propagation is possible in the region of interest but the speed of the upstream propagating waves decreases as the component of buoyancy tangential to the solid surface begins to dominate the flow, or as the edge of the surface is approached. Special attention is given to the flow under very flat surfaces, for which the effect of the tangential buoyancy force changes from negligible to dominant within a very short distance. The analysis of this case demonstrates the transition from the flow below a curved body to that below a finite horizontal flat plate.

\section{ORDERS OF MAGNITUDE AND FORMULATION}

Consider a two-dimensional body with a power law shape in a region around its bottom,

$$
z_{s}=\alpha L\left|\frac{x}{L}\right|^{2 n},
$$

where $x$ is the distance along the surface from its lowest point, $z_{s}$ is the height above this point, $L$ is a characteristic length of the body, and $n \geqslant 1 / 2$. The parameter $\alpha$ could be absorbed in the definition of $L$, but it is kept for convenience. The surface of the body is maintained at a constant temperature $T_{w}$ above the temperature $T_{\infty}$ of the surrounding fluid. Assuming that the density of this fluid is $\rho=\rho_{\infty}\left[1-\beta\left(T-T_{\infty}\right)\right]$, the boundary layer equations describing its motion at large Grashof numbers are, with the Boussinesq approximation,

$$
\begin{aligned}
& \frac{\partial u}{\partial x}+\frac{\partial v}{\partial y}=0, \\
& u \frac{\partial u}{\partial x}+v \frac{\partial u}{\partial y}=-\frac{\partial p^{\prime} / \rho_{\infty}}{\partial x}+\widetilde{g} \theta \frac{d z_{s}}{d x}+v \frac{\partial^{2} u}{\partial y^{2}}, \\
& \frac{\partial p^{\prime} / \rho_{\infty}}{\partial y}= \pm \widetilde{g} \theta, \\
& u \frac{\partial \theta}{\partial x}+v \frac{\partial \theta}{\partial y}=\frac{v}{\operatorname{Pr}} \frac{\partial^{2} \theta}{\partial y^{2}},
\end{aligned}
$$

where $y$ is the distance normal to the surface, $u$ and $v$ are the components of the velocity along and normal to the surface, $\quad \widetilde{g}=g \beta\left(T_{w}-T_{\infty}\right), \quad \theta=\left(T-T_{\infty}\right) /\left(T_{w}-T_{\infty}\right)$, $\operatorname{Pr}$ is the Prandtl number, and a $d z_{s} / d x$ $=2 n \alpha|x / L|^{2 n-1} \operatorname{sgn}(x)<1$ is understood in (3). The \pm in (4) corresponds to the boundary layers above and below the solid surface, respectively. The balance of convection, pressure gradient, and diffusion in these equations over a distance $x_{c}$ along the surface yields the well-known scales $y_{c}=x_{c} / \mathrm{Gr}_{c}^{1 / 5}, u_{c}=\sqrt{\widetilde{g} x_{c}} / \mathrm{Gr}_{c}^{1 / 10}, v_{c}=\sqrt{\widetilde{g} x_{c}} / \mathrm{Gr}_{c}^{3 / 10}$, and $\Delta p^{\prime} / \rho_{\infty}=\widetilde{g} x_{c} / \mathrm{Gr}_{c}^{1 / 5}$, with $\mathrm{Gr}_{c}=\widetilde{g} x_{c}^{3} / v^{2}$, for the boundary layer thickness, velocities, and variations of the reduced pressure, respectively (see, e.g., Gebhart et al. ${ }^{5}$ ). The characteristic length $\left(x_{c}\right)$ of the region where the pressure gradient and the tangential component of the buoyancy are comparable is then obtained by equating the orders of mag- 
TABLE I. Scaled heat flux and skin friction from the solution of (14) for several values of $n$.

\begin{tabular}{lccccccccc}
\hline & $n$ & & 0.5 & 0.7 & 1 & 1.5 & 2 & 2.5 & 3 \\
\hline$-g^{\prime}(0)$ & $(\mathrm{Pr}=0.7)$ & 0.3552 & 0.3616 & 0.3722 & 0.3862 & 0.4007 & 0.4145 & 0.4269 & 0.4674 \\
$-g^{\prime}(0)$ & $(\mathrm{Pr}=7)$ & 0.7456 & 0.7657 & 0.7928 & 0.8341 & 0.8691 & 0.9012 & 0.9304 & 1.0236 \\
$f^{\prime \prime}(0)$ & $(\mathrm{Pr}=0.7)$ & 0.9608 & 0.9135 & 0.8602 & 0.7963 & 0.7514 & 0.7169 & 0.6884 & 0.6140 \\
$f^{\prime \prime}(0)$ & $(\mathrm{Pr}=7)$ & 0.6375 & 0.6137 & 0.5853 & 0.5484 & 0.5215 & 0.4998 & 0.4820 & 0.4332 \\
\hline \hline
\end{tabular}

nitude of these two terms of (3), which yields $x_{c} L=(2 n \alpha)^{-1 /(2 n-1)} \mathrm{Gr}_{c}^{-1 / 5(2 n-1)}$, or, in terms of the Grashof number based on the characteristic size of the body $\left(\mathrm{Gr}=\widetilde{g} L^{3} / v^{2}>1\right)$,

$$
\frac{x_{c}}{L}=\frac{1}{(2 n \alpha)^{(5 / 2) /(5 n-1)} \mathrm{Gr}^{1 / 2(5 n-1)}} .
$$

The effects of variable flow properties are left out of the present formulation. These effects have been investigated by Ackroyd ${ }^{17}$ for the boundary layer above a horizontal or slightly inclined heated plate, and the conditions of applicability of the Boussinesq approximation are the same for this flow and for the problem at hand. Variable flow properties effects are surely important when accurate quantitative results are sought for in many real-world problems involving nonsmall relative temperature differences. We do not believe, however, that they would introduce qualitative changes in the steady flows discussed in this paper, and therefore, mainly for the sake of brevity and definiteness, we confine ourselves to the framework of Eqs. (2)-(5).

From this point onward the variables are referred to the previous characteristic values (with the subscript $c$ ). Denoting the nondimensional variables with the same symbols used before for their dimensional counterparts, and omitting the prime in the reduced pressure, the nondimensional boundary layer equations become

$$
\begin{aligned}
& \frac{\partial u}{\partial x}+\frac{\partial v}{\partial y}=0, \\
& u \frac{\partial u}{\partial x}+v \frac{\partial u}{\partial y}=-\frac{\partial p}{\partial x}+\theta \frac{|x|^{2 n}}{x}+\frac{\partial^{2} u}{\partial y^{2}}, \\
& \frac{\partial p}{\partial y}= \pm \theta \\
& u \frac{\partial \theta}{\partial x}+v \frac{\partial \theta}{\partial y}=\frac{1}{\operatorname{Pr}} \frac{\partial^{2} \theta}{\partial y^{2}},
\end{aligned}
$$

with the boundary conditions

$$
\begin{aligned}
& u=v=\theta-1=0 \text { at } y=0, \\
& u=p=\theta=0 \text { for } y \rightarrow \infty .
\end{aligned}
$$

In addition, we may expect the pressure gradient to become negligible compared with the tangential buoyancy force in Eq. (8) for large values of $|x|$. Then the solution would tend to the well-known self-similar solution of the simplified parabolic equations, which is of the form

$$
\psi=x|x|^{(n-1) / 2} f(\eta), \quad \theta=g(\eta),
$$

with

$$
\eta=\frac{y}{|x|^{(1-n) / 2}},
$$

where $\psi$ is the streamfunction $\left(u=\psi_{y}, v=-\psi_{x}\right.$, and $\psi=0$ at $y=0)$. Carrying these expressions into $(7)-(12)$ with $\partial p / \partial x$ left out, we find

$$
\begin{aligned}
& f^{\prime \prime \prime}+\frac{1+n}{2} f f^{\prime \prime}-n f^{2}+g=0, \\
& g^{\prime \prime}+\frac{1+n}{2} \operatorname{Pr} f g^{\prime}=0, \\
& f=f^{\prime}=g-1=0 \text { at } \eta=0, \\
& f^{\prime}=g=0 \text { for } \eta \rightarrow \infty,
\end{aligned}
$$

where the primes mean derivatives with respect to $\eta$. The solutions of (14) for different $n$ and Pr coincide with the family of exact self-similar solutions found by Braun et al. ${ }^{8}$ for special body shapes (in the present context such solutions apply for $x \gg 1$ only). The corresponding heat flux and wall shear stress are given in Table I for reference. In what follows we shall look for solutions of (7)-(12) symmetric in $x$ and with the asymptotic behavior (13).

An interesting variation of this problem occurs for shallow bowl-shaped bodies, for which the region $x=O(1)$ may cover the whole body surface and the ends of the body are encountered before the flow takes on the asymptotic form (13). We shall consider the extreme case of a finite surface with edges at $|x|=x_{e} \equiv L / x_{c}$ and with (1) holding for $|x|<x_{e}$. Now $2 L$ is the horizontal extent of the body and $\alpha<1$ is no longer a disposable parameter; $\alpha L$ being the (dimensional) height of the edges above the bottom. As can be seen from (6), the condition $x_{e}=O(1)$ is verified for $2 n \alpha=O\left(\mathrm{Gr}^{-1 / 5}\right)$, whereas the surface is effectively infinite for larger values of $n \alpha$. The resulting problem is then to solve (7)-(12) with appropriate conditions at $x= \pm x_{e}$. These conditions, and the form of the solution, are different for the flows above and below the surface. Below the surface, the flow is always directed toward the edges [Fig. 1(a)], where, as was mentioned in the introduction, the boundary layer solution develops a singularity. A summary of the local structure of the boundary layer near the edges is given in the first part of Sec. IV, in connection with the limit $n \rightarrow \infty$.

Above the surface, the boundary layer equations have multiple stationary symmetric solutions. First, solutions regular and with nonzero boundary layer thickness at $x=0$ 

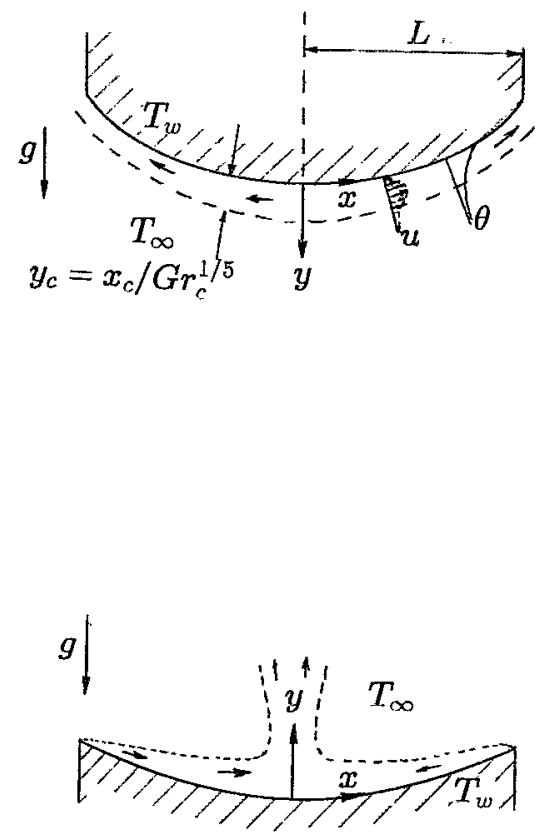

(b)

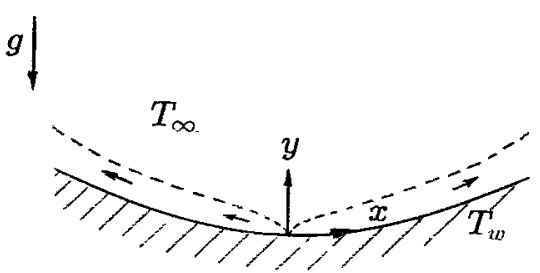

FIG. 1. Definition sketch. (a) Outbound flow below a bowl. (b) Inbound flow above a bowl. The two boundary layers collide at the center of the surface and lead to a rising plume. (c) Outbound flow above a bowl.

may exist (an example is mentioned in the following section for $n=1$ ), but they are subject to the Rayleigh-Taylor instability, which is an absolute instability in the neighborhood of $x=0$, and probably never occur. In addition, the kind of solution holding for flat horizontal plates (with the flow directed from the edges toward the center) exists also above short curved surfaces, which do not differ very much from flat plates [Fig. 1(b)]. However, the resulting downward flow may not reach $x=0$ if the body is too long $\left(\mathrm{Jones}^{16}\right)$. Instead, the boundary layer would separate and eventually generate two plumes, with the flow in the central part of the surface being directed away from the center (if a stationary boundary layer solution still applies in the central part). Another type of singular solutions of the boundary layer equations may exist for which the flow is directed everywhere away from the center, where Stewartson's solution would hold locally [Fig. 1(c)]. Still another type of boundary layer solutions would have flow directed toward the edges for $|x|$ greater than a certain value and toward the center for smaller $|x|$, with a plume rising at the center. Which of these possibilities, if any, is realized in a given situation seems to depend not only of the boundary layer equations but also on the nature of flow induced around the origin and outside the boundary layer and on stability considerations that lie beyond the scope of the present analysis. In particular, as for the problem of existence, the second and third types of solutions mentioned before, as well as the solutions for $x_{e} \rightarrow \infty$, involve boundary layers starting with zero thickness at one or more points on the surface. While this is possible as far as the boundary layer is concerned, small regions would also exist around the singular starting points where the boundary layer approximation is not applicable. Hence, the possibility of having these two types of solutions depends on whether an acceptable structure exists for the flow in such small regions, which is unlikely for smooth surfaces without edges or holes anchoring the singularities. In this paper we shall be concerned only with the boundary layer solutions sketched in Figs. 1(b) and 1(c) for the flow above a solid surface. We shall assume that the conditions at the center of the surface have been so arranged, if necessary (e.g., by applying suction through the solid surface in a small region around the center, or by suppressing half of the body), that the solution of Fig. 1(c) can be realized.

\section{RESULTS}

Equations (7)-(13) have been integrated numerically for different values of $n$ and Pr. A transient method was used for the flow below the surface, whereby time derivatives of $u$ and $\theta$ are added to the left-hand sides of (8) and (10) and the time evolution of the flow from a given initial condition $(u=\theta=0)$ is followed until it becomes stationary. For effectively infinite bodies, the computational domain extends to $|x|<x_{\infty}$, with $x_{\infty}$ sufficiently large for the pressure gradient to be negligible at the outflow boundaries. The independence of the results on $x_{\infty}$ was checked by repeating the computations with different values of this parameter. For bodies with $x_{e}$ finite, the stretched longitudinal variable $\xi_{b}=\left[1-\left(1-x / x_{e}\right)^{\varphi}\right] / \varphi$, with $\varphi<\lambda$ (see Sec. IV) was used instead of $x$, so that $\partial(u, p, \theta) / \partial \xi_{b} \rightarrow 0$ at the edge $\left(\xi_{b}=1\right)$ and conditions of extrapolation were applied there (see Ref. 13 for details).

The flow above the surface requires a different treatment. For boundary layers starting with zero thickness either at the center or at the edges of the body surface (when $x_{e}<\infty$ ), the flow near the origin of the boundary layer is given locally by Stewartson's self-similar solution, and the integration proceeds from this point onward using a marching method, after rewriting (7)-(12) in terms of the variables $\xi_{a}, y / \xi_{a}^{2 / 5}, \psi / \xi_{a}^{3 / 5}, p / \xi_{a}^{2 / 5}$, and $\theta$. Here $\xi_{a}$ is the distance from the origin of the boundary layer; i.e., $\xi_{a}=x$ or $\xi_{a}=x_{e}-x$.

Figures 2 and 3 give the nondimensional heat flux above and below infinitely long surfaces for several values of $n$ and $\operatorname{Pr}=0.7$. The dashed lines at the right of these figures correspond to the self-similar solutions (13). Above the surface (Fig. 2), the boundary layer is supposed to start at $x=0$. Then, the heat flux tends to infinity for $x \rightarrow 0$ 


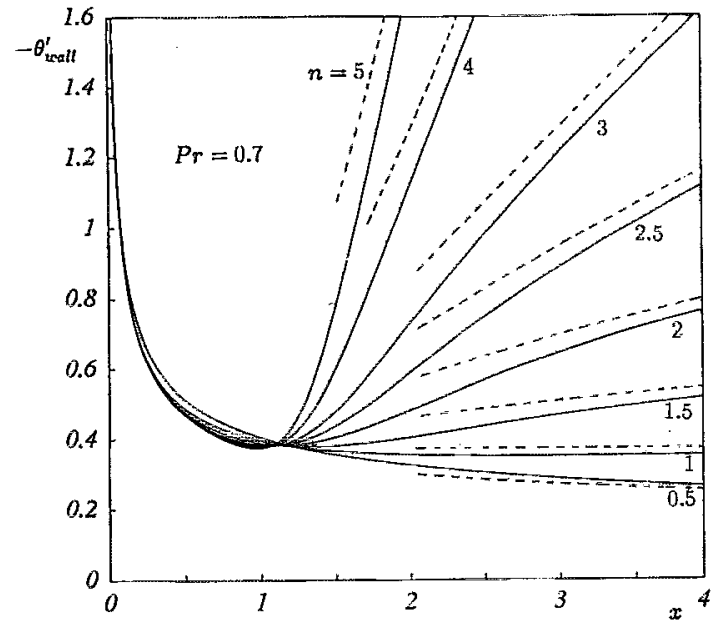

FIG. 2. Heat flux at the upper side of a solid surface as a function of the distance from the lowest point of the surface for several values of $n$ and $\operatorname{Pr}=0.7$. The dashed lines represent the asymptotic solution (13), with $-g^{\prime}(0)$ taken from Table I. The common asymptotic behavior for $x \rightarrow 0$ is that of Stewartson's solution.

in a way that does not depend on $n$ because the tangential buoyancy force is neglected in Stewartson's solution. On the contrary, the actual heat flux below the surface (Fig. 3 ) is finite at $x=0$ in all the cases (and has a peak there for $1 / 2<n<1$ ), while the self-similar solution (13) gives zero heat flux for $n>1$ and infinite heat flux for $n<1$. In the very important case $n=1$, corresponding to bodies of finite nonzero curvature radius at $x=0$, the solution of (14) gives a constant thickness boundary layer, and the pressure gradient evaluated with this solution is identically zero. This means that (13) is valid all the way down to $x=0$, both above and below the surface, for effectively infinitely long bodies. Above the surface the solution is unstable, while below the surface it provides the leading term of well-known series expansions for general body shapes (see,

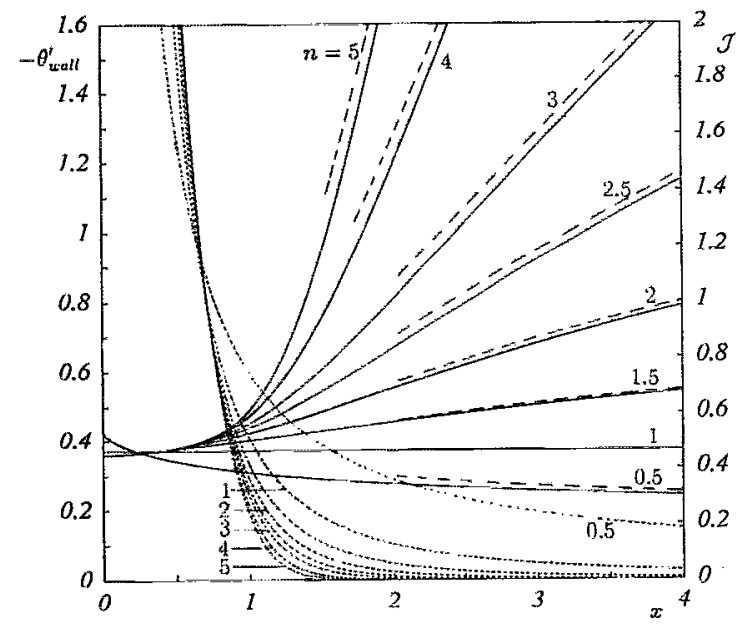

FIG. 3. Heat flux at the lower side of a solid surface as a function of the distance from the lowest point. The dashed lines are the same as in Fig. 2. The dotted lines represent the Richardson number at the wall.

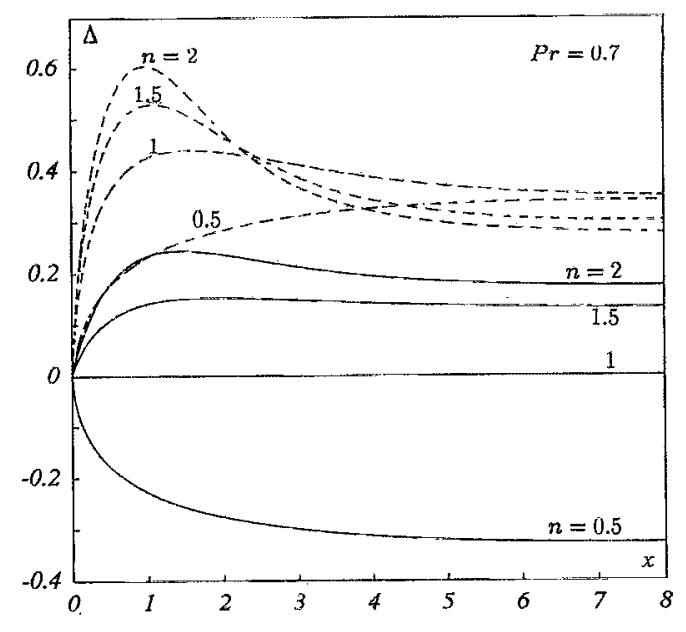

FIG. 4. Accumulated excess of the actual heat flux above that of the self-similar solution (13) as a function of the distance from the lowest point of a solid surface. Solid lines correspond to the flow below a surface and dashed lines to the flow above a surface.

e.g., Refs. 2 and 3 ). The accumulated difference between the actual heat flux and the one obtained when the solution (13) is used down to $x=0$ is

$$
\Delta(x)=2\left(-\left.\int_{0}^{x} \frac{\partial \theta}{\partial y}\right|_{0} d x+\frac{2}{1+n} g^{\prime}(0) x^{(1+n) / 2}\right),
$$

which is represented in Fig. 4 for $\operatorname{Pr}=0.7$ and four different values of $n$.

To ascertain the nature of the transition from pressure driven flow to buoyancy driven flow, we can analyze the propagation of small perturbations superposed on the stationary flow, of the form $\delta p=P(y) \exp [i k(x-c t)]$, etc., with $k$ and $c$ real and $1<k<\mathrm{Gr}^{1 / 5}$. This corresponds to wavelengths large compared with the boundary layer thickness but short compared with the length $x_{c}$ identified in (6). The following simplifications arise in the analysis of such perturbations. First, the problem is local, in the sense that the $x$ derivatives of the unperturbed velocity and temperature can be ignored compared with the much more rapid variation of the perturbation introduced at a given point. Second, these perturbations obey the linearized nonsteady form of (7)-(10) [i.e., with $\partial u / \partial t$ and $\partial \theta / \partial t$ added to the left sides of (8) and (10), respectively], but longitudinal diffusion $\left(\partial^{2} / \partial x^{2}\right)$ and vertical accelerations are still negligible [so that (9) holds as it is]. And third, transport effects are confined to a thin Stokes layer near the solid, which does not need be analyzed. Outside this layer, the linearized conservation equations for the perturbations can be combined to yield the following second-order equation for the pressure perturbation:

$$
\left(u_{b}-c\right)^{2}\left(\frac{P^{\prime}}{\theta_{b}^{\prime}}\right)^{\prime}-P=0
$$

whereas the velocity perturbation normal to the boundary layer is $V=i k\left(u_{b}-c\right) P^{\prime} / \theta_{b}^{\prime}$. Here the primes mean $y$ derivatives and the subscript $b$ denotes the unperturbed 
flow at the location $(x)$ where the perturbation is applied. Equation (15a) is to be solved with the boundary conditions

$$
P^{\prime}=0 \quad \text { at } y=0 \text { and } P \rightarrow 0 \text { for } y \rightarrow \infty
$$

(The first of these expresses the inviscid condition $V=0$ at the solid surface.) Equations (15a) and (15b) will have a nontrivial solution only for special values of $c$, and upstream perturbation propagation can occur if an eigenvalue $c<0$ exists for $x>0$ (the corresponding eigenfunction would then be regular). A term $-\left(|x|^{2 n} / i k x\right) P^{\prime}$ has been omitted in (15a); since $k \gg 1$, such a term would matter only for very large values of $|x|$. Leaving it out, the dependence of the eigenvalue problem on $x$ occurs through the functions $u_{b}$ and $\theta_{b}^{\prime}$ only. For a horizontal plate, it has been found ${ }^{13}$ that upstream propagation is possible everywhere except in the immediate vicinity of the edge, where the flow becomes critical. To investigate the existerice of negative eigenvalues for $x>0$ in the flow under a curved body, we consider the rather extreme case of the unperturbed flow (13), which holds for $x$ large. Equation (15a) rewritten in terms of $\eta, f$, and $g$ takes the form $x^{(5 n-1) / 2}\left(f^{\prime}-c\right)^{2}\left(P^{\prime} / g^{\prime}\right)^{\prime}-P=0$, where the primes mean now $\eta$ derivatives. The smallest eigenvalue of this problem, numerically computed as a function of $x$ for several values of $n$ and $\mathrm{Pr}$, is negative and decreases in modulus with increasing $x$. This means that upstream propagation is always possible for these profiles of $u_{b}$ and $\theta_{b}$ [and presumably the same is true of the actual profiles for $x=O(1)]$, but the upstream speed of the perturbations tends to zero when $x \rightarrow \infty$. A straightforward computation shows that, asymptotically, the smallest eigenvalue is

$$
c \sim-\frac{1}{f^{\prime \prime}(0) x^{(3 n-1) / 2}} \text { - for } x \gg 1 \text {. }
$$

The conclusion to be drawn from this linear analysis is that the change from elliptic to parabolic character of the stationary problem does not occur at any definite value of $x$ but only asymptotically, as $x \rightarrow \infty$.

The response of the boundary layer to a small stationary perturbation introduced at the solid wall is also of some interest. It was found elsewhere ${ }^{18}$ that the influence of such perturbation is mainly confined to a thin transport sublayer if the local Richardson number at the wall $\left(\mathscr{T} \equiv-\theta_{b}^{\prime} / u_{b}^{\prime 2}\right)$ is smaller than $1 / 4$, whereas the perturbation influences the bulk of the boundary layer if $\mathscr{T}>1 / 4$. In addition, the perturbation propagates upstream through the outer low velocity region of the boundary layer if $\operatorname{Pr}<2$, being felt ahead of the location on the wall where it was generated if the two conditions $\mathscr{T}>1 / 4$ and $\operatorname{Pr}<2$ are satisfied simultaneously. The dotted lines of Fig. 3 give the value of $\mathscr{T}$ as a function of $x$. As can be seen, $\mathscr{T}>1 / 4$ in the central region of the surface. For large $x$ (13) holds and then $T=x^{(1-5 n) / 2}$ $\times\left[-g^{\prime}(0)\right] / f^{\prime \prime}(0)^{2}$, which tends to zero for $n>1 / 5$ and to infinity for $n<1 / 5$.

Figure 5 shows the heat flux and wall shear stress

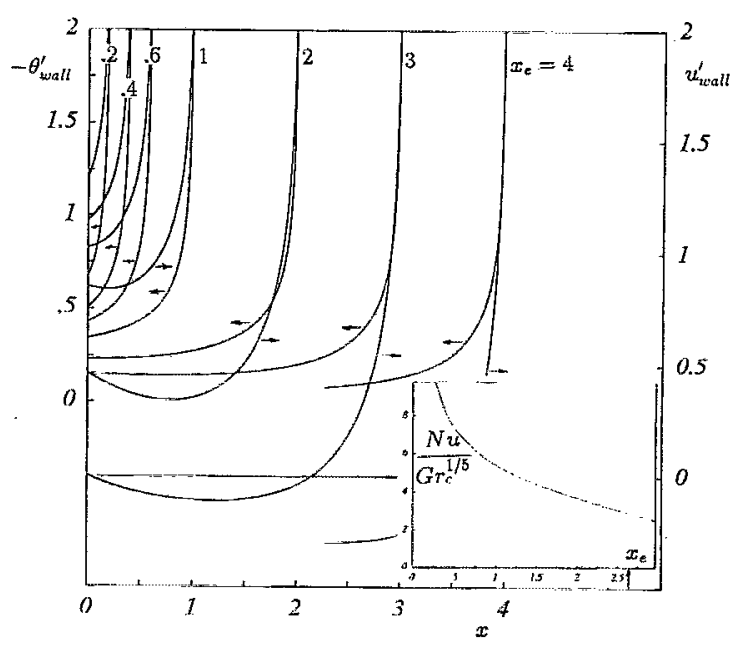

FIG. 5. Heat flux and shear stress for the inbound flow above a solid with $n=1$ and different values of $x_{e}$. Excerpt: average heat flux as a function of $x_{e}$; the dashed part corresponds to boundary layers with recirculation.

above finite length parabolic bodies $(n=1)$ with boundary layers starting at the edges for several values of $x_{e}$ (reverting to dimensional variables this means a parabolic cylinder of width much smaller than the curvature radius at its nose). A region of reverse flow at the bottom of the boundary layer appears on the central part of the surface when $x_{e}$ is greater than a certain critical value, function of $n$ and $\operatorname{Pr}$. However, as noted by Jones, ${ }^{16}$ the numerical integration can be continued through this region (the end points of the curves of Fig. 5 for $x_{e}=4$ correspond to the failure of the numerical method). A tendency to reattachment, which actually occurs for moderate values of $x_{e}$, can be observed in the figure. This is due to the decrease of the tangential component of buoyancy, which opposes the boundary layer flow, as the surface inclination diminishes near the center. The average Nusselt number, $\mathrm{Nu} / \mathrm{Gr}_{c}^{1 / 5}$ $=-\int_{0}^{x_{e}}(\partial \theta / \partial y)_{0} d x / x_{e}$, is given in the excerpt of this figure; the dashed part of the curve corresponding to boundary layers with reverse flow. A criterion on the maximum value of $x_{e}$ for which these solutions can be realized is lacking at the present time.

Last, to demonstrate the effect of the pressure gradient below finite size bodies, which, as mentioned before, enables upstream propagation of perturbations generated at some downstream location, we consider the boundary layer below a parabolic body $(n=1)$ with $x_{e}$ finite. In this case, (13) is not valid anywhere, as can be seen by noting that, owing to the symmetry, the solution would be of the form

$$
\begin{aligned}
& \psi=\sum_{m=0}^{\infty} \psi_{2 m+1}(y) x^{2 m+1}, \\
& p=\sum_{m=0}^{\infty} p_{2 m}(y) x^{2 m}, \quad \theta= \pm \sum_{m=0}^{\infty} p_{2 m}^{\prime}(y) x^{2 m},
\end{aligned}
$$

and a hierarchy of problems is obtained by inserting (17) into (7)-(12) and separating terms of like powers of $x$. The leading terms of (17) satisfy equations analogous to 


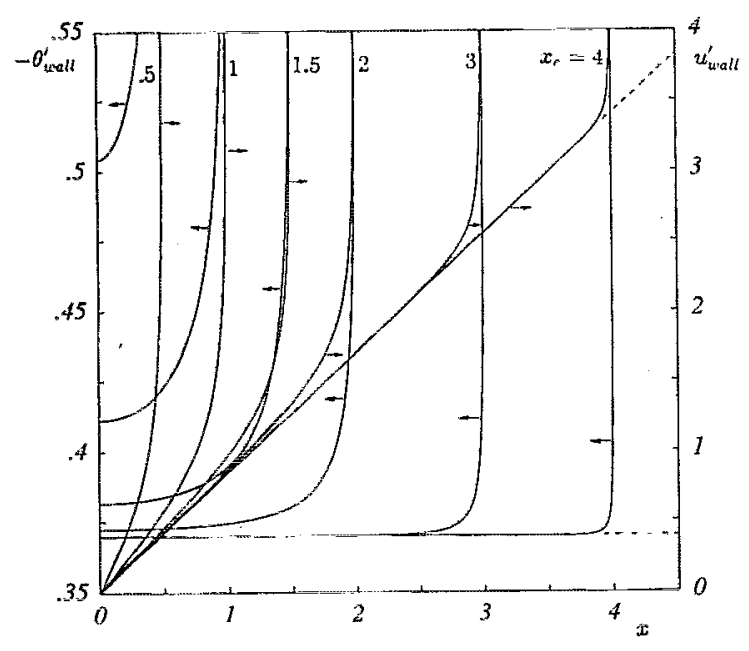

FIG. 6. Heat flux and shear stress for the outbound flow below a solid with $n=1$ and different values of $x_{e}$. The dashed lines correspond to the similarity solution (13) for an infinite parabolic cylinder.

(14), with $f=\psi_{1}, g= \pm p_{0}^{\prime}$, and $n=1$, but with the additional term $-2 p_{2}$ appearing in (14a). This $p_{2}$, which is different from zero for $x_{e}$ finite, would be determined by the next higher order problem in the hierarchy. But this problem, in turn, involves the unknown $p_{4}$, and the coupling persists for all higher orders, so that, ultimately, the location of the edges influences the solution everywhere. Numerical results for the heat flux and shear stress are presented in Fig. 6 for $\mathrm{Pr}=0.7$, together with those of the self-similar solution (13) with $n=1$, holding for an infinite parabolic cylinder. As can be seen, the influence of the edges extends to the whole boundary layer when $x_{e}$ is sufficiently small, while, as $x_{e}$ increases, the solution tends to that of the infinite parabolic cylinder except in the neighborhood of the edges.

\section{ASYMPTOTIC SOLUTION BELOW A SOLID SURFACE FOR $\boldsymbol{n}$ LARGE}

When $n$ is large, the buoyancy term $\theta|x|^{2 n} / x$ in Eq. (8) goes from negligible to dominant in short transition regions, $(|x|-1)=O[1 /(2 n-1)]$, where the character of the flow changes from pressure driven to buoyancy driven. In this section we analyze the flow in the transition region at the right half of the body for $1<(2 n-1)<\mathrm{Gr}_{c}^{1 / 5}$ [the boundary layer approximation breaks down in this region when $\left.(2 n-1)=O\left(\mathrm{Gr}_{c}^{1 / 5}\right)\right]$. As will be seen, the pressure gradient attains very large values in this region, $O(2 n-1)$, before its effect is finally overcome by that of the buoyancy. Hence, matching of the transition region and the upstream boundary layer would not be possible without a pressure gradient in the incoming flow that, with the scales used in the formulation, diverges at some apparent origin located inside the transition region in the asymptotic limit $(2 n$ $-1) \rightarrow \infty$. It turns out that this single condition determines the structure of the incoming pressure driven flow independently of its subsequent evolution. This flow has been analyzed elsewhere ${ }^{13}$ and the main features of this analysis are summarized in the following subsection as a preliminary to the discussion of the flow in the transition region.

\section{A. Incoming critical flow}

Under the action of the large pressure gradient mentioned before, transport effects become negligible in the bulk of the boundary layer approaching the transition region, being confined to a sublayer whose thickness tends to zero at the apparent origin. Denoting by $\zeta$ the distance to this origin, the flow in the inviscid region for $1 /(2 n$ $-1)<5<1$ is of the form

$$
\begin{aligned}
& u-u_{l}(y)=\xi^{\lambda} U(y)+\cdots, \quad v=\zeta^{\lambda-1} V(y)+\cdots, \\
& p-p_{l}(y)=\xi^{\lambda} P(y)+\cdots, \quad \theta-\theta_{l}(y)=\xi^{\lambda} \Theta(y)+\cdots,
\end{aligned}
$$

where $\left(u_{l}, p_{l}, \theta_{l}\right)$ are the limiting velocity, pressure, and temperature distributions at the apparent origin, and the right-hand sides of (18) represent the departures from this limiting flow. The limiting distributions depend on the evolution of the flow for $(1-x)=O(1)$, but satisfy certain criticality conditions. Thus, the limiting velocity and temperature are of the form $u_{l} \simeq A y^{\mu}$ and $\theta_{l} \simeq 1-B y^{\kappa}$ for $y<1$, with $\mu \simeq 0.364$ and $\kappa=\kappa(\mathrm{Pr})$ determined from the matching with the viscous sublayer, and matching also implies $\lambda=2 \mu /(\mu+2) \simeq 0.308$. Since $\mu<1$, the shear stress on the solid tends to infinity at the apparent origin, in keeping with the divergent pressure gradient there. The right-hand sides of (18) satisfy the conservation equations (7)-(10) linearized about the limiting distributions and with the transport and buoyancy terms omitted. Two linearly independent solutions of these equations, denoted by the subscripts $a$ and $b$ in what follows, have the following asymptotic forms for $y<1$ :

$$
\begin{aligned}
& P_{a}=1-\frac{B \kappa / A^{2}}{(\kappa+1-2 \mu)(1-2 \mu)} y^{\kappa+1-2 \mu}+\cdots, \\
& \Theta_{a}=\frac{B \kappa / A^{2}}{1-2 \mu} y^{\kappa-2 \mu}+\cdots, \\
& U_{a}=-\frac{1-\mu}{A(1-2 \mu)} y^{-\mu}+\cdots, \\
& V_{a}=-\frac{\lambda / A}{1-2 \mu} y^{1-\mu}+\cdots,
\end{aligned}
$$

and

$$
\begin{aligned}
& P_{b}=B y^{\kappa}+\cdots, \quad \Theta_{b}=-B \kappa y^{k-1}+\cdots, \\
& U_{b}=A \mu y^{\mu-1}+\cdots, \quad V_{b}=A \lambda y^{\mu}+\cdots,
\end{aligned}
$$

and, in general, only a certain linear combination of these solutions satisfies the conditions $(U, P, \Theta) \rightarrow 0$ for $y \rightarrow \infty$. As can be seen, the velocity associated with the solution (19b) diverges at the solid wall faster than the velocity associated with the solution (19a), and would therefore dominate the flow in the viscous sublayer. It turns out, however, that the problem for the viscous sublayer has no solution matching (19b) and, thus, the more rapidly divergent branch must not appear in the solution of the problem. That the solution 


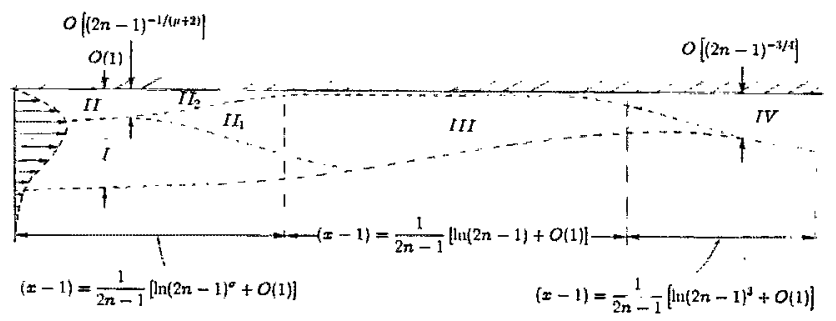

FIG. 7. Sketch of the transition region for large $n$.

(19a) alone must satisfy the boundary condition at infinity imposes a condition on the limiting distributions themselves, which is the criticality condition alluded to before.

\section{B. Transition from pressure driven to buoyancy driven flow}

Figure 7 is a sketch of the different parts of the transition region. The rapid increase of the buoyancy term in this region leads first to a splitting of the boundary layer, with viscous effects confined to the thin sublayer II. Moreover, owing to the critical character of the limiting flow, buoyancy manifest itself in a rather peculiar way: it induces a velocity perturbation in the inviscid bulk of the layer I that is much smaller than the right-hand sides of (18) but increases near the wall at the faster rate (19b), instead of (19a). In this way, the buoyancy induced velocity perturbation forces a nonlinear response of the viscous sublayer II, which, in its turn, requires a driving pressure (built up in the inviscid region I) much larger than that induced directly by the buoyancy. Then, farther downstream, region II splits into an inviscid nonlinear sublayer $\mathrm{II}_{1}$ and a viscous subsublayer $\mathrm{II}_{2}$. The first of these grows until it covers the whole boundary layer (region III), at which point the buoyancy ceases to be a small perturbation. The buoyancy force overcomes the pressure gradient shortly afterwards, leading to a decrease of the boundary layer thickness, which comes to an end when transport effects become again important (in region IV). The different stages of this transition are successively analyzed in the remainder of this section.

Let

$$
x=1+\frac{1}{2 n-1}\left[\ln (2 n-1)^{\sigma}+\xi\right],
$$

with $\xi=O(1)$ and $\sigma$ still undetermined, denote the region of nonlinear response of the viscous sublayer II. Then

$$
x^{2 n-1}=\exp [(2 n-1) \ln x] \simeq(2 n-1)^{\sigma} e^{\xi} .
$$

The solution in region $I$, outside the viscous sublayer, is of the form $u=u_{l}+U_{1}, p=p_{l}+P_{1}, \theta=\theta_{l}+\Theta_{1}$, and $v=V_{1}$, with $U_{1}, P_{1}$ and $\Theta_{1}$ small. These variables satisfy the linearized equations

$(2 n-1) \frac{\partial U_{1}}{\partial \xi}+\frac{\partial V_{1}}{\partial y}=0$

$$
(2 n-1) u_{l} \frac{\partial U_{1}}{\partial \xi}+V_{1} \frac{d u_{l}}{d y}=-(2 n-1) \frac{\partial P_{1}}{\partial \xi}+(2 n-1)^{\sigma} \theta e^{\xi}
$$

$\frac{\partial P_{1}}{\partial y}=-\Theta_{1}$,

$(2 n-1) u_{l} \frac{\partial \Theta_{1}}{\partial \xi}+V_{1} \frac{d \theta_{l}}{d y}=0$,

which can be combined into a single equation for $P_{1}$ :

$$
\frac{\partial}{\partial y}\left(\frac{\partial P_{1} / \partial y}{d \theta_{l} / d y}\right)-\frac{P_{1}}{u_{l}^{2}}+\frac{\theta_{l} / u_{l}^{2}}{(2 n-1)^{1-\sigma}} e^{\xi}=0 .
$$

This equation does not contain $\xi$ derivatives. A particular solution accounting for the nonhomogeneous buoyancy term, and its associated velocity and temperature, are

$$
\begin{gathered}
P_{p}=\frac{\theta_{e^{\xi}}}{(2 n-1)^{1-\sigma}}, \quad U_{p}=\frac{-u_{l}^{\prime} e^{\xi}}{(2 n-1)^{1-\sigma},} \\
V_{p}=(2 n-1)^{\sigma} u e^{\xi}, \quad \Theta_{p}=\frac{-\theta_{l}^{\prime} e^{\xi}}{(2 n-1)^{1-\sigma}},
\end{gathered}
$$

where the primes mean $y$ derivatives. As can be seen, the incipient effect of buoyancy on the pressure gradientdominated flow is to increase the pressure in this part of the transition region. The increase of pressure is achieved by an outward displacement of the boundary layer profiles [see (22c) and the expressions of $U_{p}$ and $\Theta_{p}$ above], leading to a deceleration of the flow in the inner part of the layer and to an acceleration in the outer part.

The general solution of $(23)$ is

$$
P_{1}=a(\xi) P_{a}(y)+b(\xi) P_{b}(y)+P_{p},
$$

and the associated velocity perturbation is

$$
U_{1}=-a(\xi)\left(\frac{u_{l}}{\theta_{l}^{\prime}} P_{a}^{\prime}\right)^{\prime}-b(\xi)\left(\frac{u_{l}}{\theta_{l}^{\prime}} P_{b}^{\prime}\right)^{\prime}+U_{p} .
$$

Here $a(\xi)$ and $b(\xi)$ are arbitrary functions and $P_{a}(y)$ and $P_{b}(\hat{y})$ are the two functions introduced in the previous subsection. On account of the critical character of the limiting flow, the boundary condition at infinity implies $b(\xi)$ $=0$. The part $U_{p}$ of the velocity has the same behavior near the wall as the solution (19b) (i.e., $y^{\mu-1}$ ), being more singular than the only acceptable solution $\left[-a\left(u_{l}^{\prime} P_{a}^{\prime} / \theta_{l}^{\prime}\right)^{\prime}=O\left(a y^{-\mu}\right)\right]$ of the homogeneous problem.

Let us consider now the flow in the viscous sublayer II. The thickness of this sublayer, determined from the balance of convection (with a velocity of order $y^{\mu}$ ) and diffusion, is of order $\delta=O\left[1 /(2 n-1)^{1 /(\mu+2)}\right]$. The velocity perturbation $U_{p}$ is of order $\delta^{\mu-1} /(2 n-1)^{1-\sigma}$ in the viscous sublayer, and the response of the sublayer becomes nonlinear when this velocity is of order $\delta^{\mu}$, which occurs for $\sigma=(\mu+1) /(\mu+2)$. A pressure of order $\delta^{2 \mu}$ is then required to influence the flow in the sublayer; this is much larger than $P_{p}$ above, and therefore the pressure gradient must come from the homogeneous solution $a(\xi) P_{a}(y)$ in (25), requiring an $a(\xi)$ of order $1 /(2 n-1)^{2 \mu /(\mu+2)}$ (the 
resulting pressure is uniform across the sublayer). Thus appropriate variables of order one in the viscous sublayer are $\xi$ and

$$
\begin{gathered}
\tilde{y}_{2}=(2 n-1)^{1 /(\mu+2)} y, \quad \widetilde{\psi}_{2}\left(\xi, \tilde{y}_{2}\right)=(2 n-1)^{(\mu+1) /(\mu+2)} \psi, \\
\tilde{\pi}(\xi)=(2 n-1)^{2 \mu /(\mu+2)} a(\xi) .
\end{gathered}
$$

In terms of these variables, the inner problem becomes

$\frac{\partial \widetilde{\psi}_{2}}{\partial \widetilde{y}_{2}} \frac{\partial^{2} \widetilde{\psi}_{2}}{\partial \xi \partial \widetilde{y}_{2}}-\frac{\partial \widetilde{\psi}_{2}}{\partial \xi} \frac{\partial^{2} \widetilde{\psi}_{2}}{\partial \widetilde{y}_{2}^{2}}=-\frac{d \tilde{\pi}}{d \xi}+\frac{\partial^{3} \widetilde{\psi}_{2}}{\partial \widetilde{y}_{2}^{3}}$,

$\widetilde{\psi}_{2}=\frac{\partial \widetilde{\psi}_{2}}{\partial \widetilde{y}_{2}}=0$ at $\tilde{y}_{2}=0$,

$\frac{\partial \tilde{\psi}_{2}}{\partial \widetilde{y}_{2}} \sim A \tilde{y}_{2}^{\mu}-\frac{1-\mu}{A(1-2 \mu)} \tilde{\pi}(\xi) \tilde{y}_{2}^{-\mu}-A \mu e^{\xi} \tilde{y}_{2}^{\mu-1}$ for $\tilde{y}_{2} \rightarrow \infty$.

The first term in (28c) matches the limiting velocity $u_{l}$; the second matches the outer velocity perturbation associated with the pressure perturbation $a(\xi) P_{a}(y)$; and the third, which represents the outward displacement of the limiting distributions, matches $U_{p}$. The unknown function $\widetilde{\pi}(\xi)$ is determined by the condition that the outward displacement be that given by the third term of $(28 \mathrm{c})$.

Upstream of the region of nonlinear response, for $(-\xi)>1$, the outward displacement becomes negligible and (28) has a solution of the form

$\widetilde{\psi}_{2}=(-\xi)^{(\mu+1) /(\mu+2)} f_{20}\left(\eta_{20}\right), \quad \tilde{\pi}=(-\xi)^{2 \mu /(\mu+2)} \tilde{\pi}_{20}$

where $\eta_{20}=\tilde{y}_{2} /(-\xi)^{1 /(\mu+2)}, \widetilde{\pi}_{20}$ is a constant, and

$$
\begin{aligned}
& (\mu+2) f_{20}^{\prime \prime \prime}+\mu f_{20}^{\prime 2}-(\mu+1) f_{20} f_{20}^{\prime \prime}+2 \mu \tilde{\pi}_{20}=0, \\
& f_{20}=f_{20}^{\prime}=0 \text { at } \eta_{20}=0, \\
& f_{20} \sim \frac{A}{\mu+1} \eta_{20}^{\mu+1}-\frac{\tilde{\pi}_{20}}{A(1-2 \mu)} \eta_{20}^{1-\mu}+0 \eta_{20}^{\mu}+\cdots \\
& \quad \text { for } \eta_{20} \rightarrow \infty .
\end{aligned}
$$

$(30 c)$

Here the constant $A$ can be absorbed by a scale transformation, rewriting (30) in terms of $A^{1 /(\mu+2)} \eta_{20}$, $f_{20} / A^{1 /(\mu+2)}$, and $\widetilde{\pi}_{20} / A^{4 /(\mu+2)}$. This asymptotic solution, and the perturbation to the limiting flow that it induces in the outer inviscid region, matches the incoming flow considered in the previous subsection. In particular, the condition that the coefficient of $\eta_{20}^{\mu}$ in (30c) be zero is fulfilled for $\mu \simeq 0.364$, as , was advanced before, and then $\tilde{\pi}_{20} / A^{4 /(\mu+2)} \simeq 0.355$.

It is worth noticing that a favorable pressure gradient $(d \tilde{\pi} / d \xi<0)$ is required to maintain the zero outward displacement of the solution of (30). Hence, the displacement becomes positive when the pressure gradient is less negative than for this solution, but no adverse pressure gradient is required to generate the outward displacement. Indeed, the numerical results (see Fig. 8) show that positive values of $d \tilde{\pi} / d \xi$ never occur.

For $\xi>1$, the thickness of the region of nonlinear response where the first and third terms on the right-hand

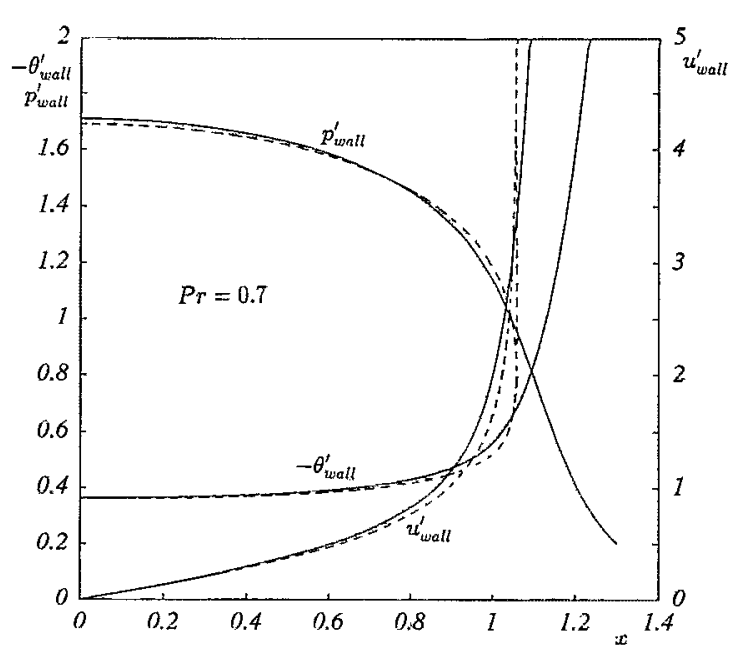

FIG. 8. Heat flux, shear stress, and pressure below a surface with $n=15$ (solid lines), and below a flat horizontal strip whose edges coincide with the inflection points of the previous pressure distribution (dashed lines).

side of (28c) are comparable (region $\mathrm{II}_{1}$ ) is ${\widetilde{y_{2}}}_{2}=O\left(e^{\bar{\xi}}\right)>1$. The velocity is of order $\vec{y}_{2}^{t}=O\left(e^{\mu \xi}\right)$, and the viscous term is negligible in the momentum equation [transport effects being confined to a thinner sub-sublayer $\mathrm{II}_{2}$ of thickness $\left.O\left(e^{-\mu \xi / 2}\right)\right]$. The balance of inertia and pressure force yields $\widetilde{\pi}=O\left(e^{2 \mu \xi}\right)$, so that the second term of (28c) remains important in $\mathrm{II}_{1}$. The solution outside the viscous subsublayer is of the form

$\widetilde{\psi}_{2}=e^{(\mu+1) \xi} f_{21}\left(\eta_{21}\right), \quad \tilde{\pi}=e^{2 \mu \xi} \widetilde{\pi}_{21}$, with $\eta_{21}=\widetilde{y}_{2} / e^{\xi}$,

where $f_{21}\left(\eta_{21}\right)$ and the constant $\widetilde{\pi}_{21}$ satisfy

$$
\begin{aligned}
& \mu f_{21}^{\prime 2}-(\mu+1) f_{21} f_{21}^{\prime \prime}+2 \mu \tilde{\pi}_{21}=0, \\
& f_{21}=0 \text { at } \eta_{21}=0, \\
& f_{21} \sim \frac{A}{\mu+1} \eta_{21}^{\mu+1}-\frac{\widetilde{\pi}_{21}}{A(1-2 \mu)} \eta_{21}^{1-\mu}-A \eta_{21}^{\mu}+\cdots \\
& \quad \text { for } \eta_{21} \rightarrow \infty .
\end{aligned}
$$

The solution of (32a) and (32b) with the asymptotic behavior given by the leading term of (32c) can be written as

$$
\eta_{21}=\int_{0}^{f_{21}} \frac{d f}{\sqrt{\left[(\mu+1) A^{1 / \mu} f\right]^{2 \mu /(\mu+1)}-2 \tilde{\pi}_{21}}} .
$$

The value of $\widetilde{\pi}_{21}$ is determined by expanding this integral for $f_{21} \gg 1$, substituting $f_{21}$ from the three-terms expansion (32c), and identifying like powers of $\eta_{21}$. The result is

$$
\tilde{\pi}_{21}=-\frac{\mu^{2 \mu} A^{2}}{2 J^{2 \mu}} \simeq-0.295 A^{2}
$$

where $J=\int_{0}^{\infty}\left[\left(1+v^{2}\right)^{-1 / 2}-v^{-1}+v^{-3} / 2\right] v^{1 / \mu} d v \simeq 0.7497$.

Nonlinearity affects the whole of the boundary layer when $\widetilde{y}_{2}=O\left[(2 n-1)^{1 /(\mu+2)}\right]$, which, according to the results above, occurs for $(2 n-1)(x-1)=\ln (2 n-1)+\xi_{3}$, with $\xi_{3}=O(1)$ (region III of Fig. 7). Appropriate independent variables to describe this region are $\xi_{3}$ and the streamfunction $\psi$, which takes the place of $y$. Leaving out 
TABLE II. Scaled heat flux and skin friction from Eq. (38) for several values of $\mathrm{Pr}$.

\begin{tabular}{ccccccccc}
\hline \hline Pr & 0.5 & 0.7 & 1 & 2 & 3 & 5 & 7 & 10 \\
\hline$-g_{3}^{\prime}(0)$ & 0.2161 & 0.2466 & 0.2823 & 036223 & 0.4158 & 0.4911 & 0.5457 & 0.6083 \\
$f_{3}^{\prime \prime}(0)$ & 1.1503 & 1.1119 & 1.0681 & 0.9754 & 0.9181 & 0.8450 & 0.7970 & 0.7467 \\
\hline \hline
\end{tabular}

a thin transport sublayer that need not be analyzed, the resulting equations, with transport effects neglected, are

$$
\begin{aligned}
& u \frac{\partial u}{\partial \xi_{3}}=-\frac{\partial p}{\partial \xi_{3}}+v_{3} \frac{\partial p}{\partial \psi}+\theta e^{\xi_{3},} \\
& \frac{\partial p}{\partial \psi}=-\frac{\theta}{u}, \\
& u \frac{\partial \theta}{\partial \xi_{3}}=0,
\end{aligned}
$$

where $v_{3}=v /(2 n-1)=u\left(\partial y / \partial \xi_{3}\right)_{\psi}$. For $\xi_{3}$ large, the velocity grows exponentially according to the law $u$ $\sim[2 \theta(\psi)]^{1 / 2} e^{\xi_{3} / 2}$, and the pressure gradient in (35a) becomes negligible. This marks the beginning of the buoyancy dominated flow. The thickness of the boundary layer decreases as $e^{-\xi_{3} / 2}$, and the transport terms, evaluated with this solution, are of order $e^{\xi_{3} / 2} /(2 n-1)$ relative to the terms displayed in (35a) and (35c). These transport terms become important to the flow in the bulk of the boundary layer when $\xi_{3}=\ln (2 n-1)^{2}+\xi_{4}$, with $\xi_{4}=O(1)$ (region IV). Then, in terms of the variables

$$
y_{4}=(2 n-1) y, \quad u_{4}=\frac{u}{2 n-1}, \quad v_{4}=\frac{v}{2 n-1}, \quad \theta,
$$

the conservation equations and boundary conditions become

$$
\begin{aligned}
& \frac{\partial u_{4}}{\partial \xi_{4}}+\frac{\partial v_{4}}{\partial y_{4}}=0, \\
& u_{4} \frac{\partial u_{4}}{\partial \xi_{4}}+v_{4} \frac{\partial u_{4}}{\partial y_{4}}=\theta e^{\xi_{4}}+\frac{\partial^{2} u_{4}}{\partial y_{4}^{2}}, \\
& u_{4} \frac{\partial \theta}{\partial \xi_{4}}+v_{4} \frac{\partial \theta}{\partial y_{4}}=\frac{1}{\operatorname{Pr}} \frac{\partial^{2} \theta}{\partial y_{4}^{2}}, \\
& u_{4}=v_{4}=\theta-1=0 \quad \text { at } y_{4}=0, \\
& u_{4}=\theta=0 \text { for } y_{4} \rightarrow \infty,
\end{aligned}
$$

which describe a purely buoyancy-driven flow. Asymptotically, for $\xi_{4}>1$, the solution in terms of the streamfunction takes the form

$$
\psi_{4}=e^{\xi_{4} / 4} f_{4}\left(\eta_{4}\right), \quad \theta=g_{4}\left(\eta_{4}\right), \quad \text { where } \eta_{4}=e^{\xi_{4}} y_{4},
$$

with

$$
\begin{aligned}
& f_{4}^{\prime \prime \prime}+\frac{f_{4} f_{4}^{\prime \prime}}{4}-\frac{f_{4}^{\prime 2}}{2}+g_{4}=0, \\
& g_{4}^{\prime \prime}+\frac{\operatorname{Pr}}{4} f_{4} g_{4}^{\prime}=0,
\end{aligned}
$$

$$
\begin{aligned}
& f_{4}=f_{4}^{\prime}=g_{4}-1=0 \text { at } \eta_{4}=0, \\
& f_{4}^{\prime}=g_{4}=0 \text { for } \eta_{4} \rightarrow \infty .
\end{aligned}
$$

This problem is the limiting form of (14) for $(2 n-1) \rightarrow \infty$ with $f=(2 n-1)^{-3 / 4} f_{4}, g=g_{4}$, and $\eta=(2 n-1)^{-1 / 4} \eta_{4}$. Results of the numerical solution of (39) for several values of $\mathrm{Pr}$ are given in Table II.

Figure 8 shows the distributions of heat flux, shear stress, and pressure on the solid below a surface with $n=15$ (solid lines), and below a flat strip ( $n$ infinite; dashed lines) whose edge coincides with the position of minimum pressure gradient in the solution for $n=15$. As can be seen, the agreement is good over most of the surface, the only differences arising in the transition region.

\section{SUMMARY}

An analysis has been carried out of the effect of the pressure gradient induced by the component of the buoyancy force normal to the surface of a curved body on the natural convection boundary layers above and below the body. The size of the region around the bottom of the body where this pressure gradient is comparable to the component of the buoyancy force tangential to the surface scales with a low power of the inverse of the Grashof number [cf. (6)], being therefore reasonably wide for values of this parameter of practical interest. Numerical solution of the relevant boundary layer equations leads to the distribution of heat flux, which, along with the boundary layer thickness, is finite at any point below a power law surface. Even though the pressure gradient effect is thought to be noticeable under realistic conditions, no detailed experiment exists, to our knowledge, with which the present results could be compared. In the very important case of body surfaces of finite nonzero curvature, to which the greatest amount of work is devoted in the literature, the pressure gradient effect is known to be weaker than for zero or infinite curvature radius, because it automatically disappears at the leading order in an expansion of the boundary layer solution around the lower stagnation point. However, the standard expansion itself may require modification for moderate Grashof numbers, as discussed at the end of Sec. III in relation with the influence of the edges of a finite parabolic cylinder, because the region where the pressure gradient effect may appear represents a large fraction of the curvature radius (about $40 \%$ for $\mathrm{Gr}=10^{5}$ ).

\section{ACKNOWLEDGMENTS}

This work was partially supported by the CICYT under projects ESP 187/90 and PB92-1075-A. 